\title{
Lenvatinib-Transarterial Chemoembolization Sequential Therapy as an Effective Treatment at Progression during Lenvatinib Therapy for Advanced Hepatocellular Carcinoma
}

\author{
Yusuke Kawamura ${ }^{a, b} \quad$ Masahiro Kobayashi ${ }^{a}$, ${ }^{b}$ Junichi Shindoh ${ }^{b, c}$ \\ Yuta Kobayashi ${ }^{b, c}$ Satoshi Okubo ${ }^{b, c}$ Licht Tominagab, d Akira Kajiwara ${ }^{a}$ b \\ Kayoko Kasuya a, b Soichi Iritani ${ }^{a, b}$ Shunichiro Fujiyama ${ }^{a}$ b Tetsuya Hosaka a, b \\ Satoshi Saitoh ${ }^{a, b}$ Hitomi Sezaki ${ }^{a, b}$ Norio Akuta ${ }^{a, b}$ Fumitaka Suzuki ${ }^{a}, b$ \\ ${\text { Yoshiyuki Suzukia, benji Ikeda }{ }^{a} \text {, }}^{a}$ Yasuji Arase ${ }^{a, b}$ Masaji Hashimotob, c \\ Tokuyo Kozukab, d Hiromitsu Kumadaa, b \\ aDepartment of Hepatology, Toranomon Hospital, Tokyo, Japan; b Okinaka Memorial Institute for \\ Medical Research, Toranomon Hospital, Tokyo, Japan; ' CHepatobiliary-Pancreatic Surgery Division, \\ Department of Gastroenterological Surgery, Toranomon Hospital, Tokyo, Japan; ${ }^{\text {dRadiation }}$ \\ Oncology Division, Department of Radiology, Toranomon Hospital, Tokyo, Japan
}

\section{Keywords}

Hepatocellular carcinoma - Lenvatinib - Oncological aggressiveness - Subsequent treatment . Lenvatinib-transarterial chemoembolization sequential therapy

\begin{abstract}
Background: The aims of this study were to evaluate the efficacy of additional treatment, especially lenvatinib-transarterial chemoembolization (TACE) sequential therapy, for unresectable hepatocellular carcinoma (HCC). Methods: Consecutive 56 patients who underwent lenvatinib treatment were reviewed. Oncological aggressiveness of tumor was estimated using a dynamic $\mathrm{CT}$ enhancement pattern classification, and clinical impact of subsequent treatment was investigated through analysis of progression-free survival (PFS), post-progression survival (PPS), and multivariate analysis of potential confounders for survival after progression during lenvatinib therapy. Results: Heterogeneous enhancement patterns (Type-3 and -4), which are reportedly associated with higher oncological aggressiveness of HCC, were associated with better objective response to lenvatinib compared to homogeneous enhancement pattern (Type-2) ( 86 and $85 \%$ vs. $53 \%$ in modified Response Evaluation Criteria in Solid Tumors), resulting in similar PFS ( $p=0.313)$. Because of significantly worse PPS, overall survival
\end{abstract}

Y. Kawamura, M. Kobayashi, and J. Shindo equally contributed to this work. 
Kawamura et al.: Lenvatinib-Transarterial Chemoembolization Sequential Therapy for Unresectable HCC

of Type-4 tumor was poor compared to Type-2 or -3 tumors $(p=0.009)$. However, subgroup of patients who achieved subsequent treatment showed significantly better PPS, regardless of CT enhancement pattern. Multivariate analysis confirmed that use of lenvatinib-TACE sequential treatment after progression during lenvatinib therapy was associated with better PPS (hazard ratio $[\mathrm{HR}], 0.08 ; 95 \% \mathrm{Cl}, 0.01-0.71 ; p=0.023$ ), while Type-4 enhancement pattern was correlated with worse PPS (HR, 2.92; $95 \% \mathrm{Cl}, 1.06-8.05 ; p=0.039)$. Conclusion: Oncological aggressiveness of HCC estimated by CT enhancement pattern was predictive of PPS after progression during lenvatinib. Successful subsequent treatment with lenvatinib-TACE sequential therapy may offer survival benefit regardless of CT enhancement pattern of HCC.

(C) 2020 The Author(s).

Published by S. Karger AG, Basel

\section{Introduction}

Hepatocellular carcinoma (HCC) is the most common type of liver cancer, which, in turn, is the third most frequent type of cancer [1]. The Barcelona Clinic Liver Cancer (BCLC) system is widely used for staging HCCs [2,3] and transarterial chemoembolization (TACE) is recommended for treatment of the intermediate stage (stage B). Meanwhile, BCLC intermediate stage includes quite heterogeneous population and can be further subclassified using the up-to-7 criteria [4] and the Child-Pugh score [5]. A recent report suggests that TACE is preferred for patients with tumors within the up-to-7 criteria and good liver function [6].

Conventionally, TACE has been performed repeatedly until the failure of the treatment. However, the efficacy of TACE remains limited even when alternative agents are used [7, 8], and many patients become TACE-refractory and their liver function declines [9] with repeat treatments. After introduction of sorafenib $[10,11]$, a worldwide trend of treatment of HCC has been switched from TACE to sorafenib before liver function declines [12-15], and the OPTIMIS study [15] has shown that such treatment strategy may extend survival outcomes. However, the efficacy of sorafenib in this setting is limited with a median overall survival (OS) $<1$ year and there remains an unmet need for new and more effective treatment strategy for advanced HCC.

With recent introduction of lenvatinib [16], encouraging results have been reported with a higher objective response rate (ORR) than that of sorafenib (18.8 vs. $6.5 \%$ in RECIST 1.1) [17]. Moreover, we have recently reported the efficacy of lenvatinib for oncologically aggressive HCC [18, 19]. Kudo et al. [20,21] reported a potential efficacy of upfront lenvatinib with subsequent TACE (lenvatinib-TACE sequential therapy) for patients with intermediate stage HCC beyond up-to-7 criteria. However, there have been no sufficient data yet on the lenvatinib-TACE sequential therapy. Therefore, this study sought to evaluate the efficacy of sequential treatment for HCC, focusing mainly on lenvatinib-TACE sequential therapy and the Type-4 enhancement pattern.

\section{Patients and Methods}

\section{Study Population}

Between October 2010 and February 2020, 92 patients received systemic anticancer treatment using lenvatinib for unresectable HCC at the Department of Hepatology, Toranomon Hospital, Tokyo, Japan. Of these, 56 patients were selected based on the following inclusion criteria: (1) unenhanced and four-phase dynamic CT study performed within 1 month prior to initiation of lenvatinib, (2) tumor with hyperenhancement in the arterial phase of dynamic CT, (3) dynamic CT study performed to evaluate the initial treatment response 2-12 weeks after initiation of lenvatinib, (4) Child-Pugh class A liver function at the time 
Kawamura et al.: Lenvatinib-Transarterial Chemoembolization Sequential Therapy for Unresectable HCC

of lenvatinib initiation, (5) BCLC stage A-C tumor(s), (6) unresectable HCC with the patient not wanting to undergo local ablation or chemoembolization therapy for various reasons (i.e., tumor size, number and location, extrahepatic metastasis, TACE refractoriness, and a variety of complications), (7) no treatment history of lenvatinib, (8) at least 1 measurable target nodule in the liver, (9) a treatment interval of $>28$ days since previous tyrosine kinase inhibitor (sorafenib or regorafenib) therapy and (10) an observation period of $\geq 4$ weeks. All the procedures were carried out in accordance with the ethical standards of the responsible committees on human experimentation (institutional and national) and with the Helsinki Declaration of 1975. The study was approved by the institutional review board of our hospital (protocol number 1438-H/ B).

\section{Diagnosis of HCC}

The diagnosis of HCC was predominantly based on the image analysis using dynamic CT. All patients underwent unenhanced and four-phase dynamic CT using a 64-multidetector CT scanner (Aquilion 64, Canon Medical Systems, Tochigi, Japan) or 80-multidetector CT scanner (Aquilion one, Canon Medical Systems) under the protocol reported elsewhere [18]. When a liver nodule showed hyperattenuation in the arterial phase and washout in the portal or delayed phase in dynamic study, the nodule was diagnosed as an HCC.

\section{Imaging Analysis of HCC and Definitions of Dynamic CT Enhancement Patterns}

Before treatment, the enhancement pattern of HCC was classified into the following 3 patterns according to our CT enhancement classification (online suppl. Fig. 1; see www.karger.com/doi/10.1159/000510299 for all online suppl. material), considering their strong relation to macroscopic classification and histopathological differentiation in nontreated HCCs [22]: Type-2, homogeneous enhancement pattern with increased arterial blood flow; Type-3, heterogeneous enhancement pattern with a septum-like structure; and Type-4, heterogeneous enhancement pattern with irregularly shaped ring structures. These unique enhancement patterns were originally established by surgically resected population, and its efficacy in prediction of oncological aggressiveness of HCC was later validated using a medical population treated by radiofrequency ablation [23].

The enhancement patterns were assessed independently by an expert hepatologist (Y. Kawamura), expert hepatobiliary surgeon (J. Shindoh), and expert radiation oncologist (L. Tominaga) who were blinded to the clinical data. Discrepancies between these 3 examiners were resolved by consensus review including an additional reviewer (K. Ikeda). All target HCC nodules appeared to be hypervascular in the study and therefore we classified all the nodules into 3 enhancement patterns (Type-2 to -4). The enhancement pattern that accounted for $70 \%$ of the nodules was defined as the predominant enhancement pattern.

\section{Lenvatinib Treatment and Assessment of Adverse Events}

Lenvatinib (Levima ${ }^{\circledR}$, Eisai, Tokyo, Japan) was administered orally to the majority of patients at a dose of either $8 \mathrm{mg}$ /day for patients $<60 \mathrm{~kg}$ or $12 \mathrm{mg} /$ day for patients $\geq 60 \mathrm{~kg}$. Treatment was discontinued when any unacceptable or serious adverse events (AEs) occurred or significantly clinical tumor progression was observed. According to the guidelines for administration of lenvatinib, the drug dose should be reduced or the treatment interrupted when a patient develops grade $\geq 3$ severe AEs or any unacceptable grade 2 drugrelated AEs occur. AEs were assessed using the National Cancer Institute's Common Terminology Criteria for Adverse Events (CTCAE), version 4.0 [24]. In accordance with the guidelines provided by the manufacturer, when a drug-related AE occurred, dose reduction or temporary interruption was maintained until the symptom resolved to either grade 1 or 2 .

\section{Treatment Protocol of Subsequent TACE Treatment after Diagnosis of the PD State}

Patients who subsequently received TACE after a diagnosis of the progressive disease (PD) state had either TACE alone or lenvatinib-TACE sequential therapy. TACE was performed on-demand according to the condition of the tumor, with the decision to continue administration of lenvatinib during the PD state based on liver function after TACE and the judgment of the physician. In patients who received lenvatinib-TACE sequential therapy, lenvatinib was discontinued for 1-14 days (median 4 days) before and 1-29 days (median 12 days) after each session of TACE, based on the condition of the patient and the tumor. At the time of readministration of lenvatinib, the starting dose was the same as that used before TACE. TACE in both groups consisted of an intra-arterial injection of lipiodol plus warmed miriplatin (Miripla ${ }^{\circledR}$, Sumitomo Dainippon Pharma Co., Ltd., Osaka, Japan), cisplatin (IA-call ${ }^{\circledR}$, Nippon Kayaku, Tokyo, Japan), and epirubicin (Farmoru-

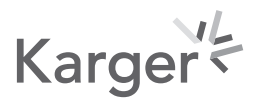


Kawamura et al.: Lenvatinib-Transarterial Chemoembolization Sequential Therapy for Unresectable HCC

bicin $^{\circledR}$, Pfizer, Tokyo, Japan). This was followed by injection of 1-mm gelatin particles (Gelpart ${ }^{\circledR}$; Nippon Kayaku) mixed with contrast agent into the blood vessel until they completely obstruct the tumor-feeding branch. In cases using miriplatin, the injector of the miriplatin/lipiodol suspension and sterilized physiological saline were placed in a container and warmed to $60^{\circ} \mathrm{C}$, followed by injection of miriplatin (60 $\mathrm{mg}$ ) suspended in $3.0 \mathrm{~mL}$ of lipiodol. The dose of miriplatin injected in each patient ranged between 50 and 100 $\mathrm{mg}$. In cases using cisplatin, at first, $100 \mathrm{mg}$ cisplatin was dissolved in $70 \mathrm{~mL}$ of saline. And then, cisplatin and lipiodol were divided into 7-10 parts, after that, 7-10 mL cisplatin solution and 0.5-1 mL lipiodol were alternately repeatedly infused. The dose of cisplatin and lipiodol injected in each patient ranged between 60 and $100 \mathrm{mg}$ in cisplatin and 3-5 mL in lipiodol. For cases using epirubicin, the agent was suspended with $2-5 \mathrm{~mL}$ lipiodol to prepare the contrast material, with 1/2-1/3 lipiodol and epirubicine (20-40 mg) used per patient. In case of lenvatinib-TACE sequential therapy, all the patients used miriplatin or epirubicin for the first subsequent TACE. Selection of the anticancer agents (miriplatin, cisplatin, or epirubicin) was decided by the investigators. On-demand TACE was repeated until the failure of treatment because of progressive major vascular invasion, disappearing tumor-feeding artery due to repeat treatment, or deteriorated hepatic function.

\section{Evaluation of Treatment Response}

The treatment response was evaluated in accordance with the modified Response Evaluation Criteria in Solid Tumors (mRECIST) [25] that was used as an auxiliary. We assessed the best tumor response during 2-12 weeks. In this study cohort, 34 of 56 patients (61\%) received dynamic CT 2-4 times to evaluate early treatment responses 2-12 weeks after lenvatinib initiation. The treatment response was assessed independently by an expert hepatologist (Y. Kawamura) and an expert hepatobiliary surgeon (J. Shindoh) who were blinded to the clinical data. Discrepancies between these 2 examiners were resolved by consensus review including an additional reviewer (K. Ikeda).

The efficacy of TACE was evaluated by dynamic CT or dynamic MRI after approximately 1-3 months based on changes in the cross-sectional area of the tumor by multiplying the major axis of the maximum cross section and the maximum diameter crossing the major axis at a right angle of viable target lesions (i.e., those showing enhancement in the arterial phase or the presence of a non-lipiodol accumulating area). The response categories were defined according to the Response Evaluation Criteria in Cancer of the Liver proposed by the Liver Cancer Study Group of Japan as treatment effect (TE) [26]. TE was defined as follows: TE4, $100 \%$ necrosis or $100 \%$ reduction in size of all targeted tumors; TE3, at least $50 \%$ or less than $100 \%$ of tumor necrotizing effect or tumor size reduction rate, respectively; TE2, effects other than TE3 or TE1; TE1, greater than $25 \%$ tumor enlargement, regardless of the necrotizing effect. We assessed the best tumor response during the 4-12 week period after TACE. TACE failure was defined as an insufficient response after $\geq 2$ consecutive TACE procedures evident on the evaluation of the response on CT or MRI after 1-3 months, even in situations where the chemotherapeutic agent had been changed and/or the feeding artery was reanalyzed. In addition, the appearance of a higher number of lesions in the liver than that recorded at the previous TACE procedure (other than the nodule being treated) was also defined as TACE failure/refractoriness [27].

\section{Follow-Up Protocol}

Physicians examined the patients every 1-2 weeks after initiation of lenvatinib, and biochemical laboratory and urine tests were also performed. After initiation of lenvatinib, the patients underwent dynamic CT to evaluate their early treatment response during the 2-12 week period. Dynamic CT or dynamic MRI was performed every 1-3 months after the first evaluation of best treatment response.

\section{Statistical Analysis}

Statistical analysis was performed using IBM SPSS software (ver. 26.0 SPSS Inc., Chicago, IL, USA). Data were expressed as the median and range. Differences in background features between each parameter were analyzed by the $\chi^{2}$ test, Fisher's exact test, Mann-Whitney U test, or Kruskal-Wallis test. $p$ values $<0.05$ were considered to indicate statistical significance. The progression-free survival (PFS) and post-progression survival (PPS) after introduction of lenvatinib were estimated by the Kaplan-Meier method, with the values compared using the log-rank test.

To identify the factors associated with PPS after initiation of lenvatinib, a multivariate analysis was performed using a Cox proportional hazards model. In the multivariate analysis, the integrated score was excluded in order to detect true factors. All factors that were at least marginally associated with PPS $(p<0.15)$ in the univariate analysis were entered into a stepwise Cox regression analysis. Significant variables were

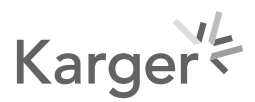


Kawamura et al.: Lenvatinib-Transarterial Chemoembolization Sequential Therapy for Unresectable HCC

Table 1. Clinical profiles and laboratory data of patients with HCC treated with lenvatinib

Patient characteristics and laboratory data

Number of patients

Gender, males: females, $n$

Age, years (range) ${ }^{\mathrm{a}}$

$\mathrm{BMI}, \mathrm{kg} / \mathrm{m}^{2}$ (range)

Body weight <60 kg: $\geq 60 \mathrm{~kg}, n$

HCV:HBV:NonB,NonC, $n$

Performance status 0:1, $n(\%)$

Platelet count, $\times 10^{3} / \mu \mathrm{L}$ (range) ${ }^{\mathrm{a}}$

Albumin, g/dL (range) ${ }^{\mathrm{a}}$

Total bilirubin, $\mathrm{mg} / \mathrm{dL}$ (range) ${ }^{\mathrm{a}}$

Prothrombin activity, \% (range) ${ }^{\mathrm{a}}$

AST, IU/L (range) ${ }^{\mathrm{a}}$

AFP, $\mu \mathrm{g} / \mathrm{L}$ (range) ${ }^{\mathrm{a}}$

DCP, AU/L (range) ${ }^{\mathrm{a}}$

Child-Pugh score 5:6, $n(\%)$

mALBI score (1:2a:2b:3), $n(\%)$

Initial dose of lenvatinib, $4 \mathrm{mg}: 8 \mathrm{mg}: 12 \mathrm{mg}, n(\%)$

Reduced starting dose of lenvatinib, $n(\%)$

History of TKI treatment, $n(\%)$

Tumor characteristics

Largest tumor diameter, $\mathrm{mm}$ (range) ${ }^{\mathrm{a}}$

Number of tumors, $n$ (range)

Macrovascular invasion, $n(\%)$

Extrahepatic metastasis, $n(\%)$

BCLC stage A:B:C, $n(\%)$

TACE failure/refractoriness, $n(\%)$

Pretreatment dynamic CT study enhancement pattern (number and ratio)

Type-2; Type-3; Type-4, $n(\%)$

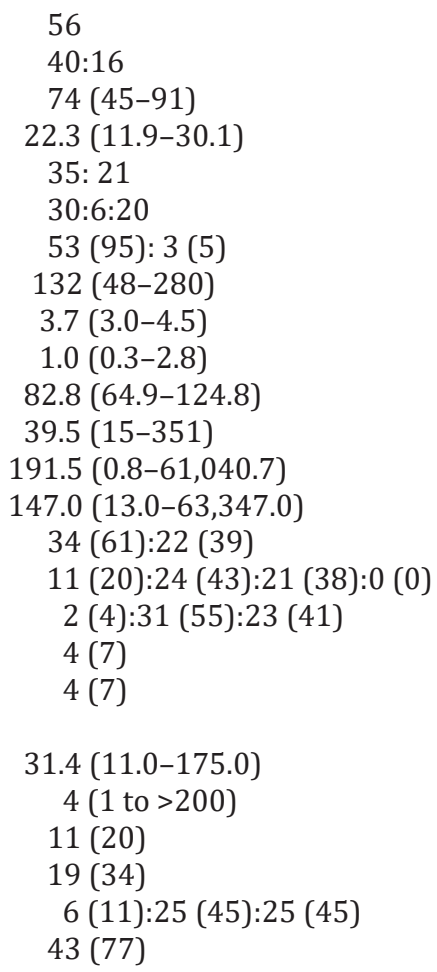

15 (27); 28 (50); 13 (23)

The composition ratio is rounded off to the first decimal place and therefore the total will not necessarily be 100. AFP, alpha-fetoprotein; BCLC, Barcelona Clinic Liver Cancer; AST, aspartate aminotransferase; DCP, des- $\gamma$-carboxyprothrombin; HBV, hepatitis B virus; HCC, hepatocellular carcinoma; HCV, hepatitis C virus; IU, international units; mALBI, modified albumin-bilirubin; NonB, NonC, neither HBV nor HCV infection present; TACE, transarterial chemoembolization; TKI, tyrosine kinase inhibitor. ${ }^{\text {a }}$ Data expressed as median (range).

selected by the stepwise method. A two-tailed $p$ value $<0.05$ was considered to be statistically significant. In addition, similar statistical analyses were performed using tumoral, general, and liver function data at the time of PD judgment in lenvatinib monotherapy. The dynamic CT enhancement pattern was affected by lenvatinib therapy; therefore, in this additional analysis, dynamic CT enhancement patters were used as the initial enhancement pattern observed before initiation of lenvatinib.

\section{Results}

\section{Overview}

Table 1 summarizes the baseline characteristics of the study population. Median age was 74 , and $40(71 \%)$ patients were male. Four patients (7\%) who were enrolled in a Global Phase II study received a higher starting dose (12 $\mathrm{mg}$ ) of lenvatinib. Median size of the largest tumor was $31.4 \mathrm{~mm}$ (range, 11-175 mm), and median number of tumors was 4 (range, 1 to $>200$ ). Of the 56 patients, $6(11 \%)$ patients with BCLC stage A disease received lenvatinib because of the location of tumor, TACE failure/refractoriness, and the patient's preference, and 25 
Table 2. Evaluation of the early treatment response after initiation of lenvatinib, grouped according to the dynamic CT enhancement pattern (Type-2-4) and analysis of imaging features using mRECIST
Kawamura et al.: Lenvatinib-Transarterial Chemoembolization Sequential Therapy for Unresectable HCC

\begin{tabular}{|c|c|c|c|c|}
\hline \multirow{2}{*}{\multicolumn{2}{|c|}{$\begin{array}{l}\text { Dynamic CT image of Response e } \\
\text { main target nodule type } \\
\text { and number }\end{array}$}} & \multicolumn{3}{|c|}{ valuation using mRECIST, $n(\%)$} \\
\hline & & & & \\
\hline \multirow[t]{3}{*}{ Type-2 $(n=15)$} & $\mathrm{CR}$ & PR & SD & PD \\
\hline & $1(7 \%)$ & $7(47 \%)$ & $6(40 \%)$ & $1(7 \%)$ \\
\hline & $\begin{array}{l}\text { ORR } \\
53 \%\end{array}$ & & & \\
\hline \multicolumn{5}{|c|}{ Heterogeneous enhancement pattern } \\
\hline \multirow[t]{3}{*}{ Type-3 $(n=28)$} & $\mathrm{CR}$ & PR & SD & PD \\
\hline & $3(11 \%)$ & $21(75 \%)$ & $2(7 \%)$ & $2(7 \%)$ \\
\hline & $\begin{array}{l}\text { ORR } \\
86 \%\end{array}$ & & & \\
\hline \multirow[t]{3}{*}{ Type $-4(n=13)$} & $\mathrm{CR}$ & PR & SD & PD \\
\hline & $2(15 \%)$ & $9(69 \%)$ & $1(8 \%)$ & $1(8 \%)$ \\
\hline & $\begin{array}{l}\text { ORR } \\
85 \%\end{array}$ & & & \\
\hline $\begin{array}{l}\text { The composition rati } \\
\text { therefore the total will } n \\
\mathrm{CT} \text {, computed tomograp } \\
\text { Response Evaluation Cri } \\
\text { rate; PD, progressive dis }\end{array}$ & $\begin{array}{l}\text { io is rounde } \\
\text { hot necessar } \\
\text { ohy; HU, Ho } \\
\text { iteria in Soli }\end{array}$ & $\begin{array}{l}d \text { off to the } \\
\text { ily be } 100 . \mathrm{C} \\
\text { unsfield uni } \\
\text { d Tumors; } 0 \\
\text { irtial respon }\end{array}$ & $\begin{array}{l}\text { first decim } \\
\text { CR, comple } \\
\text { its; mRECI } \\
\text { RR, object }\end{array}$ & $\begin{array}{l}\text { al place ar } \\
\text { te respons } \\
\text { T, Modifie } \\
\text { ve respon }\end{array}$ \\
\hline
\end{tabular}

(45\%) patients presented BCLC stage C disease (macrovascular invasion [ $n=11]$ [Vp2, $n=7$; $\mathrm{Vp} 3$ and $\mathrm{Vv} 3, n=1$; and $\mathrm{Vp} 4, n=3]$ and extrahepatic metastasis [ $n=19])$. Four patients (7\%) had a history of treatment with other tyrosine kinase inhibitors, and 43 patients $(77 \%)$ had a TACE failure/refractoriness status. The median (range) number of TACE treatments was 3 $(0-20)$ before initiation of lenvatinib. The median (range) relative dose intensity of lenvatinib was $100 \%(41-150 \%)$ at 2 weeks, $87 \%(32-150 \%)$ at 4 weeks, $74 \%(30-150 \%)$ at 8 weeks, and $70 \%(31-138 \%)$ at 12 weeks. Twenty-eight patients had died at the time of the database lock (February 18, 2020), with the median duration of lenvatinib administration being 7.5 months and the median observation period, 11.0 months.

Treatment Response and Survival Outcomes after Initiation of Lenvatinib according to the Dynamic CT Enhancement Pattern

Pretreatment CT enhancement pattern were successfully determined among the 3 independent reviewers with acceptable weighted $\kappa$ value of $0.71-0.98$. Comparison of baseline characteristics among the 3 enhancement pattern are summarized in online suppl. Table 1. In the evaluation of the early treatment response based on the dynamic CT enhancement pattern by mRECIST, the ORR of each enhancement pattern (Type-2, -3 , and -4) was 53, 86, and $85 \%$, respectively. The ORR was significantly higher with the heterogeneous enhancement pattern than with the homogeneous enhancement pattern (85 vs. 53\%, respectively) ( $p=$ 0.028) (Table 2). 
Kawamura et al.: Lenvatinib-Transarterial Chemoembolization Sequential Therapy for Unresectable HCC

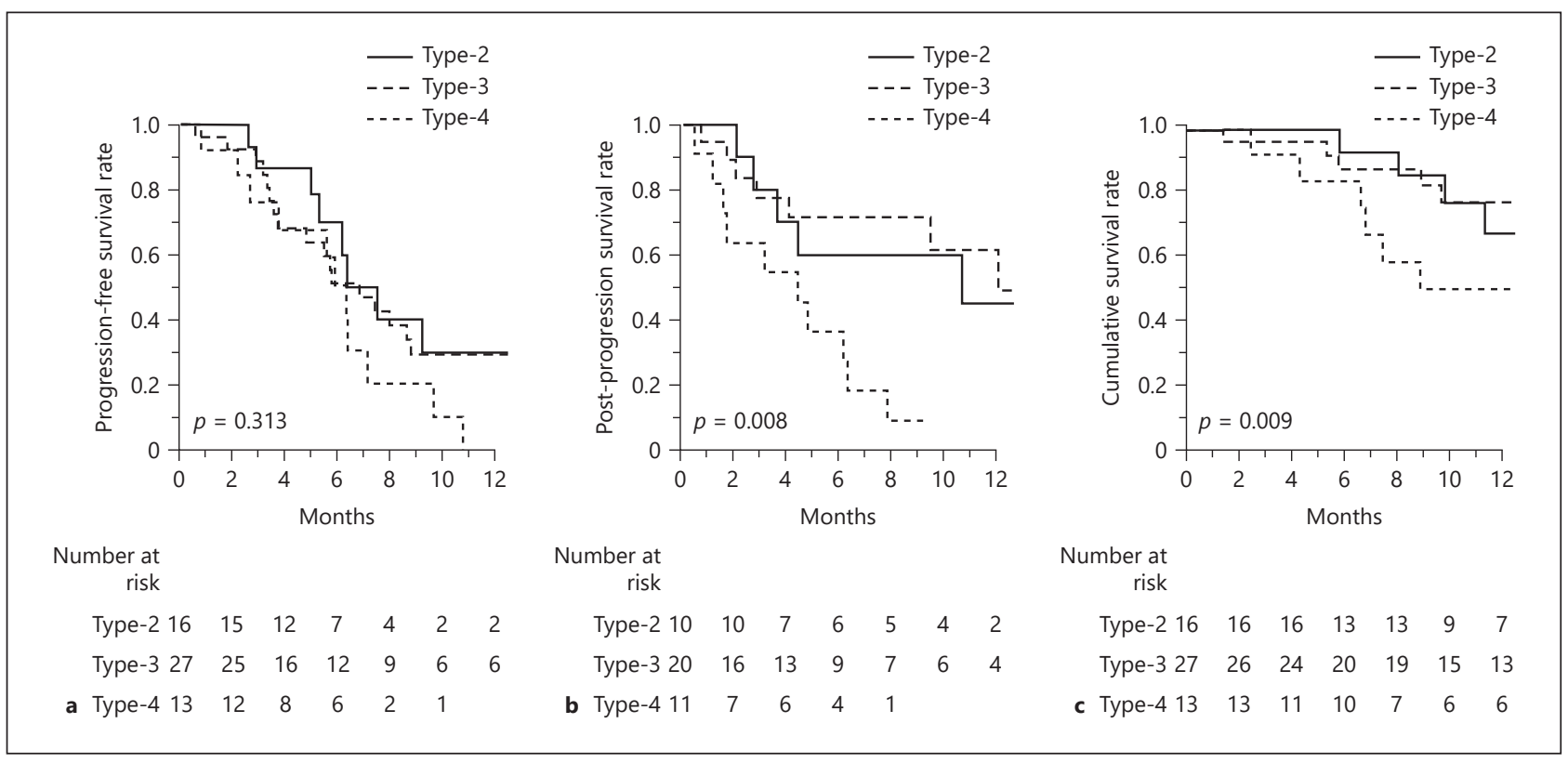

Fig. 1. Survival outcomes according to the patterns of tumor enhancement on CT scans prior to the introduction of lenvatinib. PFS rate (a), PPS rate (b), and OS rate (c). PFS, progression-free survival; PPS, post-progression survival; OS, overall survival.

Figure 1 shows the survival outcomes according to the CT enhancement pattern. Although there was no difference in PFS among the 3 groups $(p=0.313)$ (Fig. 1a), survival after progression was markedly worse when a patient presented with a Type-4 enhancement pattern before the introduction of lenvatinib ( $p=0.008)$ (Fig. 1b). As a result, cumulative survival after the introduction of lenvatinib was significantly different among the 3 groups ( $p=0.009$ ) (Fig. 1c). When the population was stratified by the BCLC stages, PFS were similar between BCLC stage A/B and stage C ( $p=0.868)$, while OS was worse in BCLC stage C, reflecting significant difference in PPS (online suppl. Fig. 2).

When looking at the difference in PPS according to the utility of subsequent treatment after in 41 patients progressed during lenvatinib therapy, the group of patients who were successfully treated with subsequent treatment (27 patients, 66\%) showed better PPS compared to the remaining patients regardless of the pretreatment CT enhancement patterns (Fig. 2). Similarly, the same tendency was confirmed when the population was subdivided by the BCLC stages as well (online suppl. Fig. 3).

\section{Details of Treatment Course and Efficacy of Subsequent TACE}

Figure 3 summarizes the details of treatment course of the 41 patients presenting with progression during lenvatinib therapy. Of the 41 patients, 21 received subsequent TACE with $(n=12)$ or without $(n=9)$ continuation of lenvatinib after diagnosis of the PD state (see online suppl. Table 2 for details of TACE treatment).

The majority of patients in the lenvatinib-TACE sequential therapy group $(n=12)$ were treated using miriplatin. There was no significant difference in the number of TACE sessions until untreatable progression between the lenvatinib-TACE sequential therapy group and the TACE monotherapy group $(n=9)(p=0.310)$. The waiting period between cancellation/interruption of lenvatinib and the first TACE session was significantly shorter in the lenvatinib- 


\begin{tabular}{l|l}
\hline Liver Cancer 2020;9:756-770 \\
\hline DOI: 10.1159/000510299 & $\begin{array}{l}\text { @ 2020 The Author(s). Published by S. Karger AG, Basel } \\
\text { www.karger.com/lic }\end{array}$ \\
\hline
\end{tabular}

Kawamura et al.: Lenvatinib-Transarterial Chemoembolization Sequential Therapy for Unresectable HCC

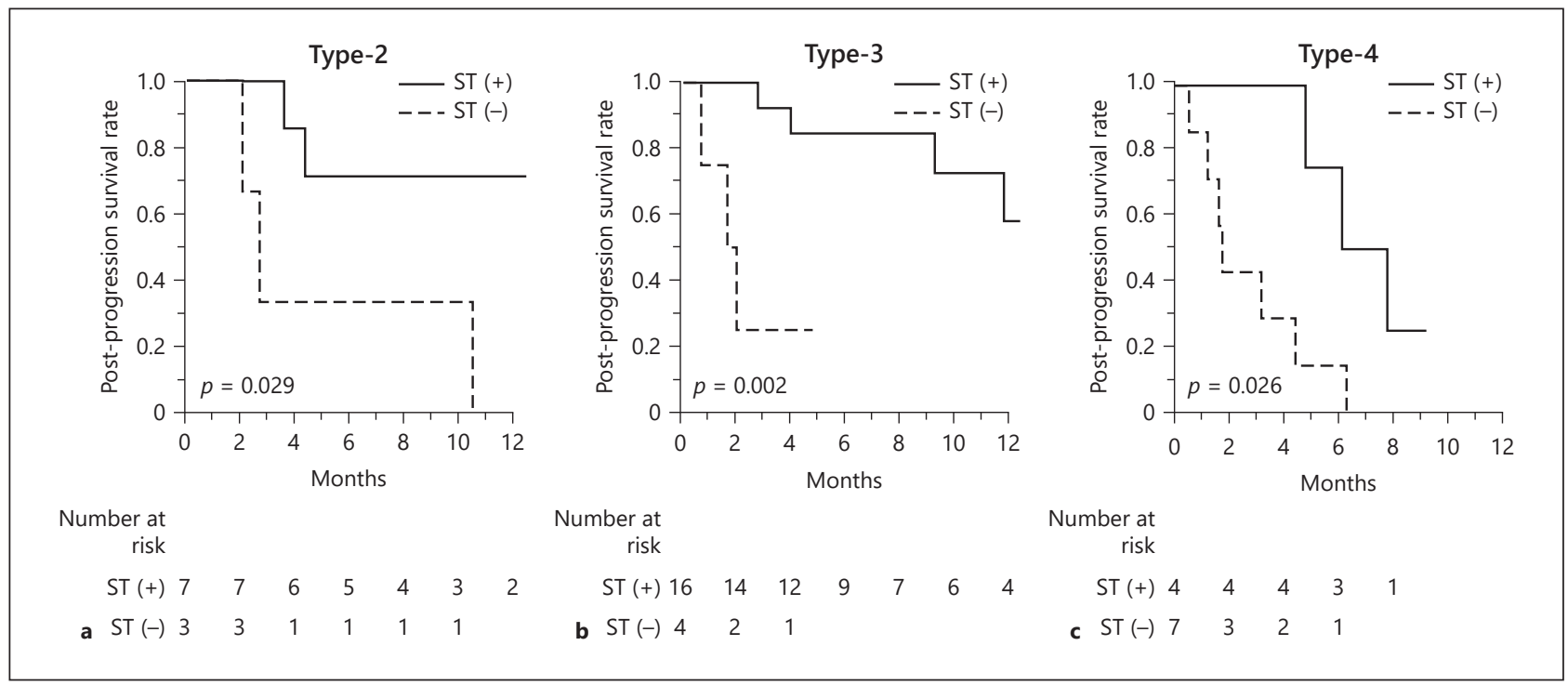

Fig. 2. PPS in lenvatinib-treated patients according to pretreatment dynamic CT, with or without subsequent treatment after diagnosis of the PD state. Type-2 enhancement pattern (a), Type-3 enhancement pattern (b), and Type-4 pattern (c). PPS, post-progression survival; ST, subsequent treatment.

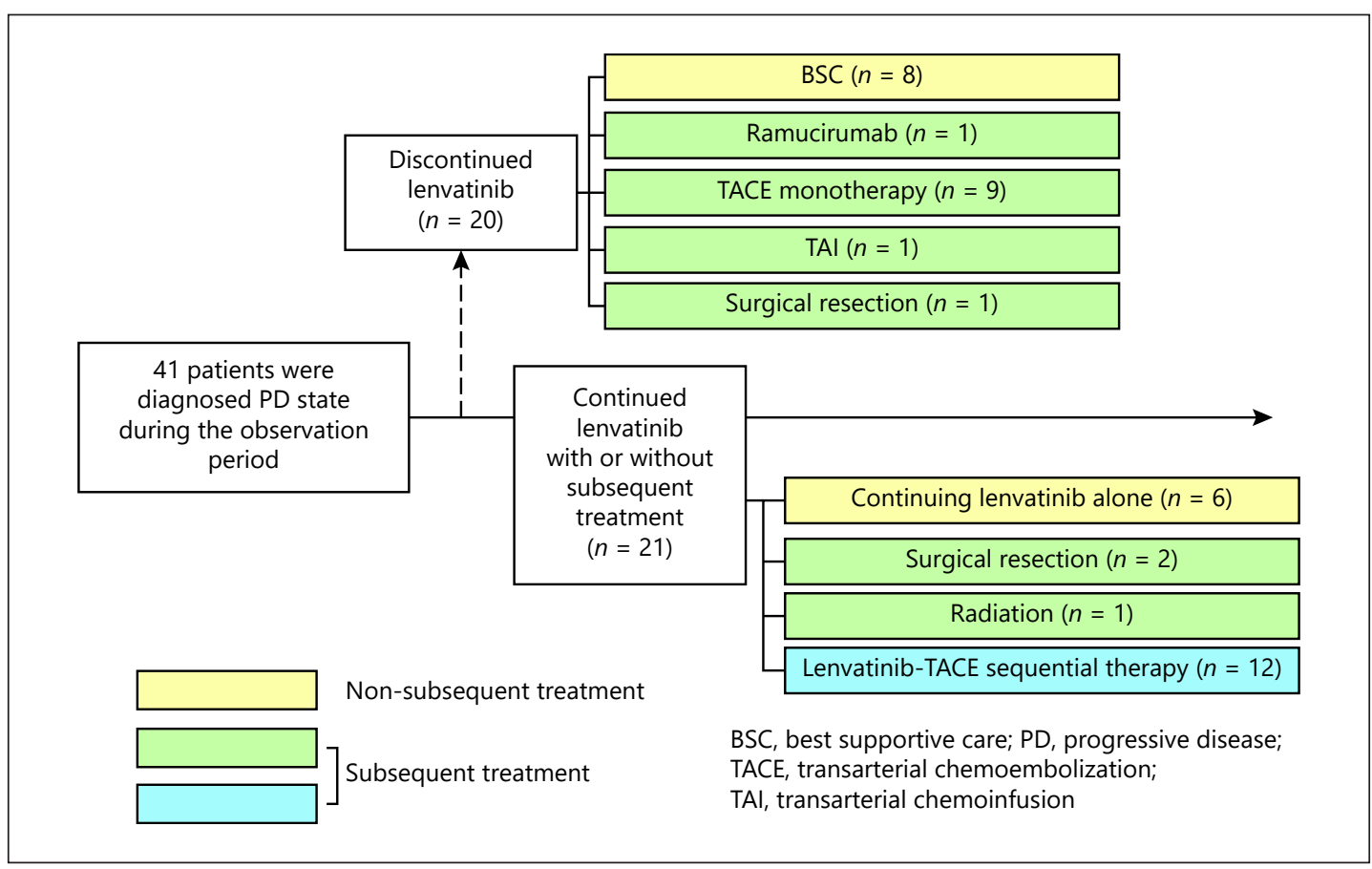

Fig. 3. Details of subsequent treatment after diagnosis of the PD state after initiation of lenvatinib treatment.

TACE sequential therapy ( $p=0.018)$. There was no significant difference in the initial treatment effect between these 2 subgroups: TACE monotherapy group (TE4, $n=3$ [33\%]; TE3, $n=1$ [11\%]; TE2, $n=3$ [33\%]; TE1 $n=2$ [22\%]) and lenvatinib-TACE sequential therapy group (TE4, $n=3$ [25\%]; TE3, $n=5$ [42\%]; TE2, $n=3$ [25\%]; TE1, $n=1$ [8\%]) ( $p=0.839)$. 
Table 3. Predictive factors for PPS

\begin{tabular}{|c|c|c|c|c|c|c|}
\hline & $p$ value $^{\mathrm{a}}$ & Coefficients $^{\mathrm{b}}$ & SE & Wald $\chi^{2}$ & HR & $95 \% \mathrm{CI}$ \\
\hline \multicolumn{7}{|l|}{ Heterogeneous Type-4 CT enhancement pattern } \\
\hline prior to introduction of lenvatinib & 0.039 & 1.070 & 0.519 & 4.26 & 2.92 & $1.06-8.05$ \\
\hline Tumor number +1 nodule & 0.001 & 0.016 & 0.005 & 10.97 & 1.02 & $1.01-1.03$ \\
\hline $\mathrm{AFP}+100 \mu \mathrm{g} / \mathrm{L}$ & 0.001 & 0.009 & 0.003 & 10.60 & 1.01 & $1.00-1.02$ \\
\hline \multicolumn{7}{|l|}{ Subsequent treatment after PD state } \\
\hline Other subsequent treatment & 0.207 & -0.740 & 0.586 & 1.59 & 0.48 & $0.15-1.51$ \\
\hline Lenvatinib-TACE sequential therapy & 0.023 & -2.515 & 1.110 & 5.13 & 0.08 & $0.01-0.71$ \\
\hline
\end{tabular}

Multivariate Cox regression was applied using stepwise backward selection. Of the potential predictors, factors presenting marginal association $(p<0.15)$ with PPS after the introduction of lenvatinib in univariate analysis were included in the initial model. Then factors that showed no or limited statistically significant association $(p>0.1)$ adjusted for the remaining factors in the model were deleted from the model in a stepwise fashion. The 19 variables tested were as follows ( $p$ values in univariate analysis): age (0.323), gender (0.424), BMI (0.649), etiology (HCV vs. others) (0.384), serum albumin (0.010), serum total bilirubin (0.922), prothrombin activity (0.839), platelet count (0.007), serum aspartate aminotransferase (0.161), serum AFP (0.013), plasma des-gamma carboxyprothrombin (0.002), tumor diameter $(<0.001)$, tumor number $(<0.001)$, macrovascular invasion (0.006), extrahepatic metastasis (0.004), heterogeneous type-4 CT enhancement pattern prior to treatment (0.004), TACE failure/refractoriness (0.176), reduced starting dose of lenvatinib (0.977), and subsequent treatment after diagnosis of PD state (other subsequent treatment 0.001 and lenvatinib-TACE sequential therapy 0.001). PPS, post-progression survival; AFP, alpha-fetoprotein; 95\% CI, 95\% confidence interval; CT, computed tomography; HR, hazard ratio; PD, progressive disease; SE, standard error; TACE, transarterial chemoembolization. ${ }^{\text {a }}$ Based on the likelihood test adjusted for the other factors in the final model. ${ }^{\mathrm{b}}$ Estimated coefficient for the variable and the associated standard error.

None of the patients developed a major complication after lenvatinib-TACE sequential therapy other than the post-embolization syndrome, which was mild or equal in severity to that observed with TACE monotherapy.

\section{Predictors of PPS after Introduction of Lenvatinib}

Table 3 summarizes the results of multivariate analysis for PPS during lenvatinib therapy using pretreatment variables. Of the 19 tested variables, Type-4 CT enhancement pattern (hazard ratio [HR], 2.92; 95\% CI, 1.06-8.05; $p=0.039)$, tumor number (HR, 1.02; 95\% CI, 1.01-1.03; $p=0.001$ ), AFP level (HR, 1.01; 95\% CI, 1.00-1.02; $p=0.001$ ) were significantly associated with poor PPS. Lenvatinib-TACE sequential therapy was associated with better PPS (HR, 0.08; 95\% CI, 0.01-0.71; $p=0.023$ ). Adjusted PPS curves showed clear differences according to selection of subsequent treatment after progression during lenvatinib therapy (Fig. 4).

When comparing the clinical variables according to the selection of subsequent treatment, patients who were successfully treated with lenvatinib-TACE sequential therapy showed tendency toward lower des-gamma carboxyprothrombin levels, higher proportion of ChildPugh A patients, lower number of tumor nodules, and smaller size of tumor (Table 4). However, when using clinical data at the time of progression, multivariate analysis confirms similar results as follows: Type-4 CT enhancement pattern (HR, 6.11; 95\% CI, 1.78-21.03; $p=$ 0.004), number of tumor (HR, 1.02; 95\% CI, 1.01-1.04; $p<0.001$ ), AFP level (HR, 1.02; 95\% CI, 1.01-1.03; $p<0.001$ ), and lenvatinib-TACE sequential therapy (HR, 0.08; 95\% CI, 0.01$0.68 ; p=0.021$ ) (see online suppl. Table 3; online suppl. Fig. 4).

\section{Karger ${ }^{\prime \prime}=$}


Kawamura et al.: Lenvatinib-Transarterial Chemoembolization Sequential Therapy for Unresectable HCC

Fig. 4. Adjusted PPS curves grouped according to subsequent treatment with lenvatinib after diagnosis of the PD state. PPS, post-progression survival.

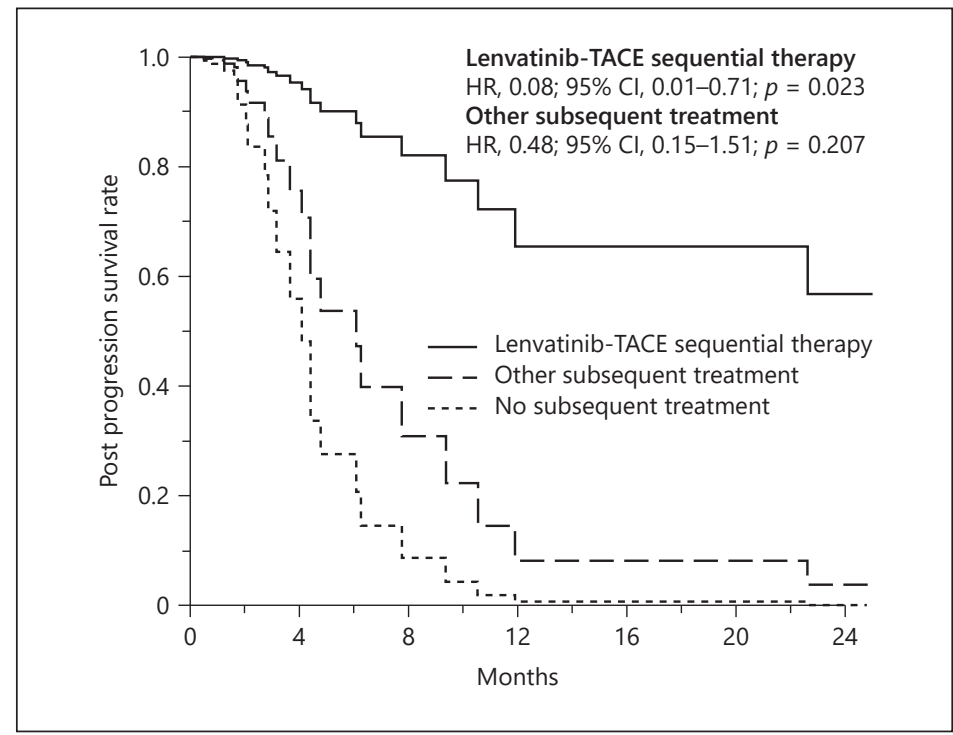

\section{Discussion}

As we reported previously [18], this study confirms that heterogeneous enhancement pattern (i.e., Type-3 and -4) were correlated with higher response to lenvatinib (Table 2) and showed similar PFS compared to homogeneous enhancement pattern (i.e., Type-2), while Type-4 tumor was associated with significantly worse PPS, resulting in shorter OS (Fig. 1). Given that heterogeneous enhancement pattern is strongly correlated with worse macroscopic subtype of HCC (simple nodular with peripheral growth or multinodular patterns) and poor differentiation, the present results suggest that PPS is predominantly dependent on the malignant potential of tumor and might not be prolonged even when objective response is achieved with lenvatinib. However, this study also confirms that successful subsequent treatment may be advantageous to improve PPS regardless of malignant potential of HCC which is estimated by the CT enhancement pattern (Fig. 2). Therefore, we have extensively investigated the clinical factors, especially the efficacy of subsequent treatment, to improve PPS among population undergoing lenvatinib treatment.

In this study we performed a multivariate analysis to identify the factors that predicted PPS, including subsequent treatment after diagnosis of the PD state. In general, we should perform these analyses using only pretreatment data. However, it is well known that the majority of patients who receive lenvatinib experience disease progression in the relatively early phase of this treatment. Therefore we need to consider various subsequent treatments after confirmation of the PD state in patients who have sufficient residual liver function and can receive radiofrequency ablation, surgical resection, radiation therapy, other molecular target agents, or TACE with or without lenvatinib before the initiation of lenvatinib. In addition, the adjusted PPS curves showed a clear difference when lenvatinib was used according to the presence of subsequent treatment after diagnosis of the PD state (Fig. 4).

Moreover, subsequent treatment showed a useful treatment effect regardless of the estimated tumor differentiation (Fig. 2), with lenvatinib-TACE sequential therapy being the most effective subsequent treatment after diagnosis of the PD state. We also performed an additional analysis that examined tumoral, general, and liver function data collected at the time of PD judgment. Similarly, the results of a multivariate analysis showed lenvatinib-TACE sequential therapy was the most effective subsequent treatment after diagnosis of the PD state. Indeed, it has been considered that unfavorable macroscopic subtypes and poor differentiation of HCC are 
Kawamura et al.: Lenvatinib-Transarterial Chemoembolization Sequential Therapy for Unresectable HCC

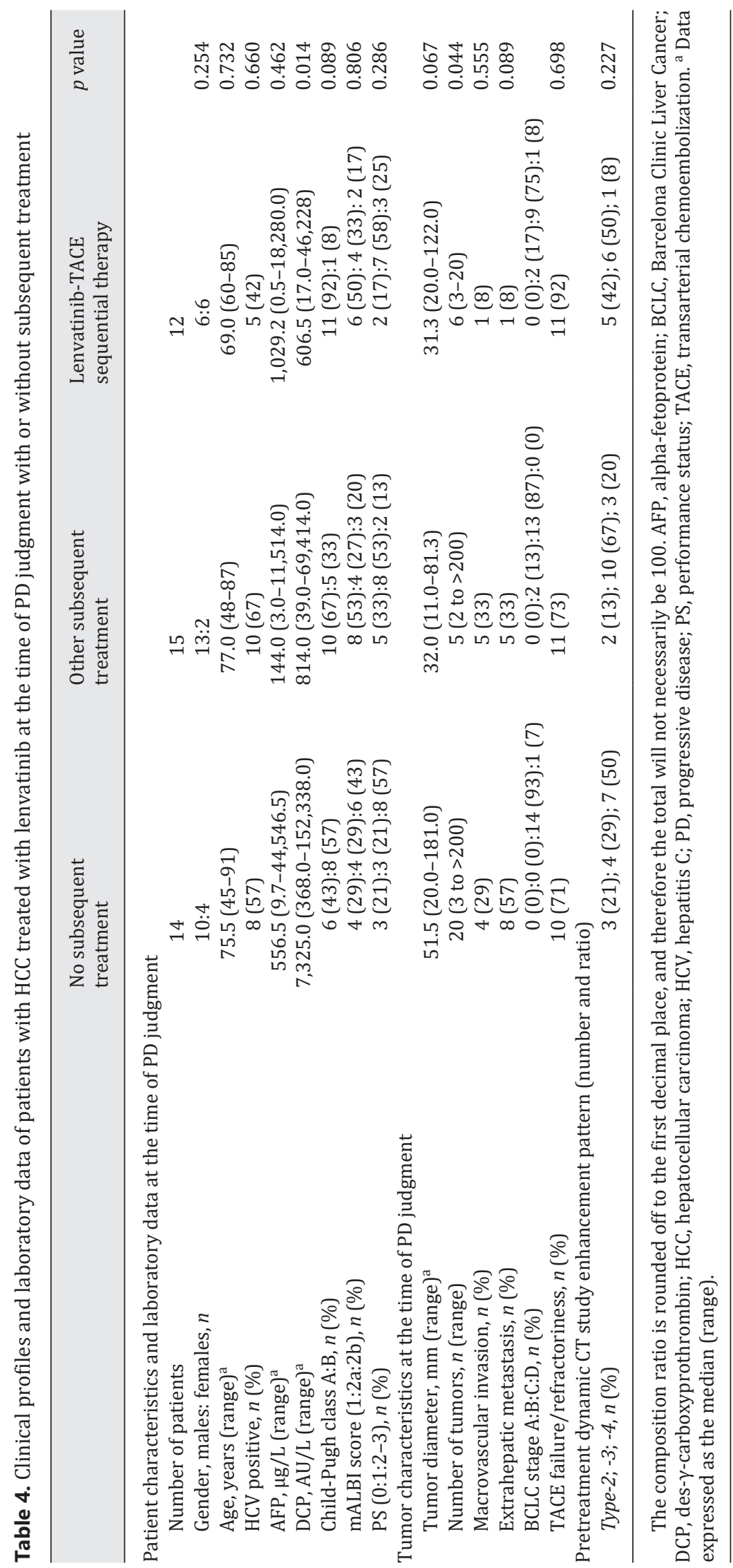


not suitable for TACE [28]. Given the efficacy of lenvatinib-TACE sequential therapy for such oncologically unfavorable findings of HCC, however, it could be a potential treatment option for HCCs, regardless of the macroscopic subtypes or tumor differentiation of HCC. Therefore, the most important clinical message in this clinical study is as follows: it is necessary to consider lenvatinib-TACE sequential therapy as one of the first-line subsequent treatments when a patient has an intrahepatic target nodule, and it is important to control treatment intensity in order to maintain sufficient residual liver function at the time of diagnosis of the PD state. On the other hand, only 1 patient in the study received a second-line molecular-target agent (ramucirumab) after diagnosis of the PD state. In fact, ramucirumab was only recently established as a secondline treatment. However, various molecular target agents have been developed as second-line treatment for sorafenib $[29,30]$. Therefore, we do not have sufficient clinical data of these molecular target agents as second-line treatment for lenvatinib. Because of this clinical background, many other subsequent treatments were used in the study cohort.

Recently, the efficacy of sorafenib-TACE sequential therapy for HCC was reported [31]. As Jain [32] reported previously, normalization of tumor vasculature with antiangiogenic therapy may be a potential mechanisms of improved treatment outcomes in cancers. We have also experienced significant regression of large tumor vessels for bulky HCC during lenvatinib treatment among the present cohort (online suppl. Fig. 5). Although the majority of patients in the lenvatinib-TACE sequential therapy group had already presented refractoriness to TACE in their clinical course (Table 4), dense and uniform lipiodol accumulation was observed in $75 \%$ of patients immediately after subsequent TACE, with $67 \%$ of patients acquiring TE3 or a higher therapeutic effect (online suppl. Table 2). Therefore, we speculate that regression of tumor vessels with administration of lenvatinib may be one of the factors that contribute to the improved treatment effect of lenvatinib-TACE sequential therapy.

The majority of the cases examined in this study were post-marketing cases, so long-term analysis has not yet been completed. In the present analysis, we have small number of patients who were converted to surgery during lenvatinib therapy. Although the mid-term surgical outcomes remain inconclusive due to relatively short observational period and general impression of survival outcomes of $\mathrm{R} 2$ resection cases was not satisfactory, we have experienced 2 long survivors who achieved R0 resection as a subsequent treatment for progression after lenvatinib therapy. Therefore, future studies are needed to further clarify the impact of each treatment options at progression during lenvatinib treatment.

Limitations of the present study include its retrospective nature and single-center study for relatively small number of series. Also, median follow-up period (11.0 months) was relatively short compared to that of the global phase III REFLECT trial (27.7 months) [17]. Furthermore, various subsequent treatments were used because of no established treatment strategy in the new era of lenvatinib, and it is difficult to clarify the actual impact of each treatment when number of cases are limited. Therefore, future study using larger, multicenter cohorts with sufficient observational period is needed to validate the present outcomes. Additionally, the major limitation of the present study is a lack of pathological data. However, the dynamic CT enhancement pattern used in the present study was originally established by comparison with pathological data in surgical population, and PPS and OS were actually significantly different according to the enhancement patterns determined by 3 independent reviewers as we confirmed in Figure 1. The present results validated that the CT enhancement criteria may also be useful in prediction of oncological aggressiveness of tumor in untreated population and recurrent HCCs, in compatible with our previous work [18]. Given that HCC shows histological heterogeneity in each nodule, and it is not realistic to perform a tumor biopsy of all target nodules to confirm histological differentiation in actual clinical settings, the present outcomes confirm that the CT enhancement pattern can be used as a substitute for estimation of oncological aggressiveness of HCC in medical population. Regarding the

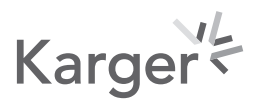


choice of subsequent treatment, some patients could not shift to lenvatinib-TACE sequential therapy because of a decrease in their hepatic functional reserve during treatment, and this could be an issue of clinical management even if lenvatinib-TACE sequential treatment might offer beneficial effects in survival. It would be necessary to consider optimal timing and strategy in future clinical studies, as well.

\section{Conclusion}

The heterogeneous Type-4 enhancement pattern of HCC was associated with a poor survival outcome, especially in PPS after lenvatinib treatment. However, the most important clinical information presented here is that subsequent treatment, especially lenvatinib-TACE sequential therapy, may offer clinical benefits on PPS regardless of estimated tumor differentiation at the time of progression during lenvatinib therapy.

\section{Acknowledgements}

This work was supported in part by grants from the Ministry of Health, Labour and Welfare in Japan and Japan Agency for Medical Research and Development.

\section{Statement of Ethics}

This retrospective nonintervention study was approved by the Institutional Review Board, Toranomon Hospital (protocol number 1438-H/B). The study was performed in accordance with the Declaration of Helsinki. Because of the anonymous nature of the data and the opt out disclosed on our institution's homepage (https://www.crc-toranomonhosp.jp/wp-content/uploads/2020/01/rinken_1438HB_2.pdf), the requirement for additional informed consent to participate in this study was deemed unnecessary according to the Japanese national regulation "Ethical Guidelines for Medical and Health Research Involving Human Subjects" (https://www.mhlw.go.jp/file/06-Seisakujouhou-10600000-Daijinkanboukouseikagakuka/0000080278. pdf).

\section{Conflict of Interest Statement}

Yusuke Kawamura, MD, PhD reports receiving a honoraria from Eisai. Masahiro Kobayashi, MD. reports receiving a honoraria from Eisai. Junichi Shindoh, MD, PhD reports receiving a honoraria from Eisai. Hiromitsu Kumada, MD, PhD reports receiving a honoraria from Eisai. The other authors declare no conflicts of interest.

\section{Funding Sources}

Okinaka Memorial Institute for Medical Research and the Japanese Ministry of Health, Labour and Welfare.

\section{Author Contributions}

Yusuke Kawamura, MD, PhD - study concept and design, acquisition of data, statistical analysis, and drafting of the manuscript. Masahiro Kobayashi, MD - acquisition of data and statistical analysis. Junichi Shindoh, MD, PhD - acquisition of data, statistical analysis, and critical revision of the manuscript. Yuta Kobayashi, MD - acquisition of data. Satoshi Okubo, MD, PhD - acquisition of data. Licht Tominaga, MD, $\mathrm{PhD}$ - acquisition of data and statistical analysis. Akira Kajiwara, MD - acquisition of data. Kayoko Kasuya, 
Kawamura et al.: Lenvatinib-Transarterial Chemoembolization Sequential Therapy for Unresectable HCC

MD - acquisition of data. Soichi Iritani, MD, PhD - acquisition of data. Shunichiro Fujiyama, MD - acquisition of data. Tetsuya Hosaka, MD - acquisition of data. Satoshi Saitoh, MD - acquisition of data. Hitomi Sezaki, MD - acquisition of data. Norio Akuta, MD, PhD - acquisition of data. Fumitaka Suzuki, MD, PhD - acquisition of data. Yoshiyuki Suzuki, MD, PhD - acquisition of data. Kenji Ikeda, MD, PhD - acquisition of data, statistical analysis, and study supervision. Yasuji Arase, MD, PhD - acquisition of data. Masaji Hashimoto, MD, PhD acquisition of data. Takuyo Kozuka, MD, PhD - acquisition of data. Hiromitsu Kumada, MD, PhD - acquisition of data. All authors had access to the data and participated in the writing of this manuscript.

\section{References}

1 Ferlay J, Soerjomataram I, Dikshit R, Eser S, Mathers C, Rebelo M, et al. Cancer incidence and mortality worldwide: sources, methods and major patterns in GLOBOCAN 2012. Int J Cancer. 2015;136(5):E359-86.

2 Forner A, Reig ME, de Lope CR, Bruix J. Current strategy for staging and treatment: the BCLC update and future prospects. Semin Liver Dis. 2010;30(1):61-74.

3 Forner A, Reig M, Bruix J. Hepatocellular carcinoma. Lancet. 2018;391(10127):1301-14.

4 Mazzaferro V, Llovet JM, Miceli R, Bhoori S, Schiavo M, Mariani L, et al. Predicting survival after liver transplantation in patients with hepatocellular carcinoma beyond the Milan criteria: a retrospective, exploratory analysis. Lancet Oncol. 2009;10(1):35-43.

5 Pugh RN, Murray-Lyon IM, Dawson JL, Pietroni MC, Williams R. Transection of the oesophagus for bleeding oesophageal varices. Br J Surg. 1973;60(8):646-9.

6 Bolondi L, Burroughs A, Dufour JF, Galle PR, Mazzaferro V, Piscaglia F, et al. Heterogeneity of patients with intermediate (BCLC B) hepatocellular carcinoma: proposal for a subclassification to facilitate treatment decisions. Semin Liver Dis. 2012;32(4):348-59.

7 Kawamura Y, Ikeda K, Hirakawa M, Hosaka T, Kobayashi M, Saitoh S, et al. Efficacy of platinum analogue for advanced hepatocellular carcinoma unresponsive to transcatheter arterial chemoembolization with epirubicin. Hepatol Res. 2009;39(4):346-54.

8 Seki A, Hori S. Switching the loaded agent from epirubicin to cisplatin: salvage transcatheter arterial chemoembolization with drug-eluting microspheres for unresectable hepatocellular carcinoma. Cardiovasc Intervent Radiol. 2012;35(3):555-62.

9 Hiraoka A, Kumada T, Kudo M, Hirooka M, Koizumi Y, Hiasa Y, et al. Hepatic function during repeated TACE procedures and prognosis after introducing sorafenib in patients with unresectable hepatocellular carcinoma: multicenter analysis. Dig Dis. 2017;35(6):602-10.

10 Llovet JM, Ricci S, Mazzaferro V, Hilgard P, Gane E, Blanc JF, et al. Sorafenib in advanced hepatocellular carcinoma. N Engl J Med. 2008;359(4):378-90.

11 Cheng AL, Kang YK, Chen Z, Tsao CJ, Qin S, Kim JS, et al. Efficacy and safety of sorafenib in patients in the AsiaPacific region with advanced hepatocellular carcinoma: a phase III randomised, double-blind, placebocontrolled trial. Lancet Oncol. 2009;10(1):25-34.

12 Ogasawara S, Chiba T, Ooka Y, Kanogawa N, Motoyama T, Suzuki E, et al. Efficacy of sorafenib in intermediatestage hepatocellular carcinoma patients refractory to transarterial chemoembolization. Oncology. 2014; 87(6):330-41.

13 Arizumi T, Ueshima K, Minami T, Kono M, Chishina H, Takita M, et al. Effectiveness of sorafenib in patients with transcatheter arterial chemoembolization (TACE) refractory and intermediate-stage hepatocellular carcinoma. Liver Cancer. 2015;4(4):253-62.

14 Ohki T, Kondo M, Karasawa Y, Kawamura S, Maeshima S, Kojima K, et al. Evaluation of the efficacy of sorafenib on overall survival in patients with hepatocellular carcinoma using FT rate: a devised index. Adv Ther. 2017; 34:1097-108.

15 Peck-Radosavljevic M, Kudo M, Raoul J-L, Lee HC, Decaens T, Heo J, et al. Outcomes of patients (pts) with hepatocellular carcinoma (HCC) treated with transarterial chemoembolization (TACE): global OPTIMIS final analysis. J Clin Oncol. 2018;36(15_Suppl):4018.

16 Ikeda K, Kudo M, Kawazoe S, Osaki Y, Ikeda M, Okusaka T, et al. Phase 2 study of lenvatinib in patients with advanced hepatocellular carcinoma. J Gastroenterol. 2017;52(4):512-9.

17 Kudo M, Finn RS, Qin S, Han K-H, Ikeda K, Piscaglia F, et al. Lenvatinib versus sorafenib in first-line treatment of patients with unresectable hepatocellular carcinoma: a randomised phase 3 non-inferiority trial. Lancet. 2018;391(10126):1163-73.

18 Kawamura Y, Kobayashi M, Shindoh J, Kobayashi Y, Kasuya K, Sano T, et al. Pretreatment heterogeneous enhancement pattern of hepatocellular carcinoma may be a useful new predictor of early response to lenvatinib and overall prognosis. Liver Cancer. 2020 Jun;9(3):275-92.

19 Kawamura Y, Kobayashi M, Shindoh J, Kobayashi Y, Kasuya K, Sano T, et al. 18F-fluorodeoxyglucose uptake in hepatocellular carcinoma as a useful predictor of an extremely rapid response to lenvatinib. Liver Cancer. 2020;9(1):84-92.

20 Kudo M, Ueshima K, Chan S, Minami T, Chishina H, Aoki T, et al. Lenvatinib as an initial treatment in patients with intermediate-stage hepatocellular carcinoma beyond up-to-seven criteria and Child-Pugh A liver function: a proof-of-concept study. Cancers. 2019;11(8):11. 
21 Kudo M. A new treatment option for intermediate-stage hepatocellular carcinoma with high tumor burden: initial lenvatinib therapy with subsequent selective TACE. Liver Cancer. 2019;8(5):299-311.

22 Kawamura Y, Ikeda K, Hirakawa M, Yatsuji H, Sezaki H, Hosaka T, et al. New classification of dynamic computed tomography images predictive of malignant characteristics of hepatocellular carcinoma. Hepatol Res. 2010; 40(10):1006-14.

23 Kawamura Y, Ikeda K, Seko Y, Hosaka T, Kobayashi M, Saitoh S, et al. Heterogeneous type 4 enhancement of hepatocellular carcinoma on dynamic CT is associated with tumor recurrence after radiofrequency ablation. AJR Am J Roentgenol. 2011;197(4):W665-73.

24 National Cancer Institute. Division of Cancer Treatment and Diagnosis. Cancer therapy evaluation program. Adverse events/CTCAE [accessed 2018 Dec 23]. Available from: https://ctep.cancer.gov/protocolDevelopment/electronic_applications/ctc.htm\#ctc_40.

25 Lencioni R, Llovet JM. Modified RECIST (mRECIST) assessment for hepatocellular carcinoma. Semin Liver Dis. 2010;30(1):52-60.

26 Kudo M, Ueshima K, Kubo S, Sakamoto M, Tanaka M, Ikai I, et al., Liver Cancer Study Group of Japan. Response Evaluation Criteria in Cancer of the Liver (RECICL) (2015 Revised version). Hepatol Res. 2016;46(1):3-9.

27 Kudo M, Matsui O, Izumi N, Kadoya M, Okusaka T, Miyayama S, et al. Transarterial chemoembolization failure/ refractoriness: JSH-LCSGJ criteria 2014 update. Oncology. 2014;87(Suppl 1):22-31.

28 Kudo M, Han K-H, Ye S-L, Zhou J, Huang Y-H, Lin S-M, et al. A changing paradigm for the treatment of intermediate-stage hepatocellular carcinoma: Asia-Pacific primary liver cancer expert consensus statements. Liver Cancer. 2020:1-16.

29 Bruix J, Qin S, Merle P, Granito A, Huang YH, Bodoky G, et al. Regorafenib for patients with hepatocellular carcinoma who progressed on sorafenib treatment (RESORCE): a randomised, double-blind, placebocontrolled, phase 3 trial. Lancet. 2017;389(10064):56-66.

30 Zhu AX, Kang Y-K, Yen C-J, Finn RS, Galle PR, Llovet JM, et al. Ramucirumab after sorafenib in patients with advanced hepatocellular carcinoma and increased $\alpha$-fetoprotein concentrations (REACH-2): a randomised, double-blind, placebo-controlled, phase 3 trial. Lancet Oncol. 2019;20:282-96.

31 Kudo M, Ueshima K, Ikeda M, Torimura T, Tanabe N, Aikata H, et al. Randomised, multicentre prospective trial of transarterial chemoembolisation (TACE) plus sorafenib as compared with TACE alone in patients with hepatocellular carcinoma: TACTICS trial. Gut. 2020;69(8):1492-501.

32 Jain RK. Normalization of tumor vasculature: an emerging concept in antiangiogenic therapy. Science. 2005; 307(5706):58-62. 